\title{
La geometría, eje integrador del pensamiento matemático en educación básica
}

\author{
Nelsy Rocío González" \\ Marisol Chavarro Tulcán ** \\ Carlos Mojica Manrique **** \\ Clara Yarley Peña Murcia ${ }^{* * * * *}$
}

Artículo de investigación

Fecha de Recepción: 2 julio 2018.

Fecha de Aprobación: 2 noviembre 2018.

\section{Resumen}

El presente artículo presenta resultados generales y avances de la primera etapa de una propuesta de intervención en aula a través de la investigación acción educativa con enfoque cualitativo. El objetivo central de la investigación es el de potenciar el pensamiento matemático en la educación básica de la Institución Educativa Técnica y Académica Antonio Nariño del municipio de Villa de Leyva - Boyacá, desde una visión geométrica contextualizada. Se tendrán como referentes, estándares básicos de competencias, malla curricular vigente en el área de matemáticas, derechos básicos de aprendizaje (DBA), resultados obtenidos del análisis de los procesos de enseñanza y aprendizaje de las matemáticas y la incorporación de rúbricas de desempeño en el aula; autores como Zoltan Dienes, con el uso de material manipulativo en el aula de matemáticas, Van Hiele, con el desarrollo del pensamiento geométrico, y David Ausubel, en el aprendizaje significativo, sirven de sustento al marco teórico de la investigación.

Palabras clave: Geometría, rendimeinto escolar, material didáctico, enseñanza de las matemáticas, aprendizaje, formación básica.
* Universidad Pedagógica y Tecnológica de Colombia Boyacá - Colombia nelsy.gonzalez@uptc.edu. co

** Institución Educativa Técnica y Académica Antonio Nariño - Boyacá Colombia marisolchavarro. mc@gmail.com

*** Institución Educativa

Técnica y Académica

Antonio Nariño - Boyacá

- Colombia ingecarmo@ gmail.com

**** Institución Educativa

Técnica y Académica

Antonio Nariño - Boyacá

- Colombia claris1581@ gmail.com

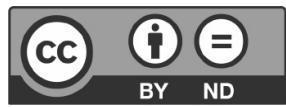




\section{Introducción}

Desde el área de matemáticas de la Institución Educativa Técnica y Académica Antonio Nariño, en el último lustro y de manera sistemática, se ha evidenciado un desempeño general por debajo de lo esperado, tanto en el rendimiento de los estudiantes (tazas de reprobación), como en las pruebas estandarizadas.

La experiencia de los autores de la investigación da indicios de que las razones por las cuales esto ocurre en el aula se relacionan, tanto con el desempeño, como con las didácticas usadas para abordar los contenidos establecidos en las mallas curriculares que la Institución ha implementado. Además, al analizar la Autoevaluación Institucional, el Plan de Mejoramiento Institucional y los resultados en el Índice Sintético de Calidad Educativa ISCE, los resultados invitan a buscar soluciones a las problemáticas anteriormente expuestas con nuevas propuestas que permitan generar cambios que trasciendan y puedan ser adoptados por la institución educativa y vayan a la vanguardia con las competencias evaluadas en las pruebas internas y externas: comunicación, representación y modelación, razonamiento y argumentación, planteamiento y resolución de problemas en los grados $3^{\circ}, 5^{\circ}, 7^{\circ}$ y $9^{\circ}$, y en el grado $11^{\circ}$ : interpretación y representación; formulación $\mathrm{y}$ ejecución, $\mathrm{y}$ razonamiento $\mathrm{y}$ argumentación. Por consiguiente, el presente trabajo busca elaborar e implementar una propuesta didáctica en el aula que permita potenciar el pensamiento matemático en la educación de básica, desde una visión geométrica contextualizada abordando las aportaciones teóricas de Zoltan Dienes, Carlos Eduardo Vasco y del modelo Van Hiele, teniendo en cuenta las teorías cognitivas de Jean Piaget, Lev Vygotsky y de aprendizaje significativo de David Ausubel.

Para el desarrollo de la propuesta se tendrán en cuenta tres objetivos específicos determinados de la siguiente manera: primero, caracterizar socio-demográficamente los niños y niñas que cursan los grados tercero, quinto y sexto de básica de los grupos focalizados, analizando el desarrollo de los procesos de enseñanza aprendizaje de las matemáticas. Lo anterior se determinará como referente para el segundo objetivo: diseñar la propuesta metodológica basada en las mallas curriculares manejadas en la institución, los estándares básicos de competencias, Derechos Básicos de Aprendizaje (DBA), resultados obtenidos del análisis de los procesos de enseñanza y aprendizaje de las matemáticas y rúbricas de desempeño con el fin de clarificar lo que se espera del trabajo del estudiante, de valorar su ejecución y de facilitar la realimentación para el tercer periodo académico de 2017 en los grados focalizados y, por último, el análisis y la interpretación de resultados en la aplicación de los instrumentos conlleva al tercer objetivo que es la elaboración de la propuesta final, que permite contribuir al mejoramiento del pensamiento matemático desde la visión geométrica en contexto. 


\section{Referentes teóricos}

\section{Antecedentes}

Dentro de los hallazgos previos de la presente investigación, se han encontrado diferentes estudios los cuales han dado una visión para el desarrollo del mismo. Inicialmente se encontró en el año 2003 una guía de seis cuadernillos de matemática titulados Tareas que promuevan el razonamiento en el aula a través de la geometría por Carmen Samper de Caicedo, Leonor Camargo, Uribe Cecilia Leguizamón de Bernal, miembros de la Asociación Colombiana de Matemáticas y docentes de la Universidad Nacional de Colombia. Este libro es una alternativa didáctica para la enseñanza de la geometría, innovando en el aula a través de distintas actividades que permiten la adquisición de conceptos y el aprendizaje de la misma, fundamentándose en diferentes teorías y estudios investigativos la cual ha sido de gran aporte ya que da una visión aún más didáctica del cómo enseñar geometría a través de estrategias que van a la vanguardia con la educación colombiana.

Por otra parte, también se consultó el trabajo de grado en modalidad de maestría elaborado en el año $2009 \mathrm{La}$ enseñanza de la geometría con fundamento en la solución de problemas cotidianos, una propuesta metodológica orientada por el modelo de Van Hiele, aplicada a estudiantes de la I.E Rafael J. Mejía del municipio de Sabaneta (Ant.), presentado por Wilson Alonso Piedrahita Restrepo, Jesús Antonio Londoño Ramírez y Elkin
Uribe Múnera, como requisito a grado de Magister en Educación y Desarrollo Humano Línea de Ambientes Educativos que se dicta en la Universidad de Manizales - Centro Internacional de Educación y Desarrollo Humano. Este trabajo se fundamentó en la búsqueda de respuestas a las exigencias encontradas en el aula con respecto a los ambientes de aprendizaje, con el fin de solucionar los problemas de aprendizaje cotidianos y facilitando el aprendizaje significativo, dando respuesta a los mismos desde su contexto. Este trabajo brinda para la investigación una mirada contextualizada importante para reconsiderar las estrategias didácticas que permitan al estudiante potencializar sus conocimientos y la comprensión de los mismos. Este trabajo fue fundamentado en la teoría de Van Hiele para el aprendizaje y la enseñanza de la geometría.

Leonor Camargo, docente del Departamento de Matemáticas de la Universidad Pedagógica Nacional Martín Acosta y docente de la Escuela de Matemáticas de la Universidad Industrial de Santander, publicó en la revista Tecné, Episteme y Didaxis. Su artículo muestra un análisis de diferentes teorías sobre la enseñanza de la geometría desde la etapa infantil hasta la universitaria, luego se centra en el modelo de Vinner del aprendizaje de conceptos matemáticos aún más especifica que la de Hiele, ya que son compatibles y su fuerte son las representaciones gráficas, reflexionando sobre estas y basándose en la definición de imágenes conceptuales y definiciones conceptuales para mostrar el papel que
Leonor Camargo, docente del Departamento de Matemáticas de la Universidad Pedagógica Nacional Martín Acosta y docente de la Escuela de Matemáticas de la Universidad Industrial de Santander, publicó en la revista Tecné, Episteme y Didaxis. 
Apoyados en los referentes, para la investigación se presenta el marco teórico estructurado en tres categorías principales: teorías cognitivas, pensamiento matemático y pensamiento geométrico. cumple el análisis crítico de ejemplos y contraejemplos en la comprensión y aprendizaje de los estudiantes y el uso que el docente dé a las mismas.

También se encontró el artículo publicado en el año 2011 en las Memorias del $20^{\circ}$ Encuentro de Geometría y sus Aplicaciones, realizado en la Universidad Pedagógica Nacional en Bogotá Colombia, presentado por Ángel Gutiérrez y denominado "Reflexiones sobre la enseñanza de la geometría en los niveles de primaria y secundaria”. En él se realiza una introducción a los diferentes elementos didácticos que se usan en la enseñanza de la geometría en los diferentes niveles educativos. Al igual que los anteriores trabajos, se centra en el modelo de Van Hiele considerándolo el más efectivo en la organización de las diferentes etapas y contenidos de la enseñanza de la geometría.

Por último, se localizó un artículo publicado en la revista Comunicación, Lenguaje y Educación, escrito por Carlos Vasco en el año 1990 titulado "Las matemáticas elementales como proceso condicionado por la cultura". En él se examina "el mito" de que "La matemática es un lenguaje universal". Vasco concluye que el aprendizaje de la matemática no solo depende del aprendizaje de la cultura si no de la historia y la literatura, también que la mayoría de los docentes suponen que los estudiantes no saben nada de matemática al ingresar por primera vez a la escuela, por tanto, la matemática moderna puede llegar solo a un pequeño grupo de niños; Vasco aduce que solo los niños que pueden construir sus propios conceptos y, a pesar de lo que crean sus maestros, son inmunes a la "fobia de matemática", sin embargo, Vasco con su experiencia concluye que están ligadas a la cultura tanto como otras asignaturas y elabora una nueva teoría general de sistemas, descrita en este documento, que implica una serie de consecuencias pedagógicas que pueden ser adaptadas a una cultura y añade un sin número de pautas para la enseñanza de la teoría de conjuntos, lógica, números naturales, enteros, fracciones y geometría.

\section{Marco teórico}

Apoyados en los referentes, para la investigación se presenta el marco teórico estructurado en tres categorías principales: teorías cognitivas, pensamiento matemático y pensamiento geométrico.

\section{Teorias cognitivas}

Las teorías cognitivas del aprendizaje abordadas son: Teoría del desarrollo cognitivo de Piaget (Ochaíta, 1983); del Aprendizaje significativo de Ausubel (Ausubel, Novak y Hanesian, 1983); del desarrollo cognitivo mediante interacción social de Vygotsky (Baquero, 1996), teorías que contribuyen a la justificación y dan los medios necesarios para brindar a los estudiantes enseñanzas que permiten construir el conocimiento y la comprensión, a través de un proceso secuencial de exploración y razonamiento deductivo e inductivo. Las teorías constructivistas estudiadas 
se basan en la creencia de que los niños construyen su propio conocimiento y comprensión conceptual a través de su propia actividad.

Por su parte, Piaget fue el primero en introducir el concepto de niveles de aprendizaje: él creía que el progreso de un nivel al siguiente se debía a cambios biológicos y que el nivel superior era innato y se alcanzaba cuando los estudiantes se dieran cuenta de ello. Teniendoencuentasus teorías, el papeldel profesor es establecer un ambiente para permitir que los estudiantes construyan este conocimiento matemático. Este entorno proporcionaría a los estudiantes oportunidades de formular hipótesis, probar su pensamiento, manipular materiales y comunicar su comprensión con el fin de construir conocimiento matemático. El trabajo piagetiano (Ochaíta, 1983) tiene dos temas principales:

Primero, nuestra representación mental del espacio no es una "lectura" perceptiva de lo que nos rodea, más bien, lo construimos a partir de nuestra representación mental, de nuestro mundo a través de la reorganización progresiva de nuestra manipulación activa anterior de ese ambiente.

Segundo, la organización progresiva de las ideas geométricas sigue un orden definido y este orden es más experiencial (y posiblemente más matemáticamente lógico) que histórico. Es decir, inicialmente se construyen relaciones topológicas. En estas se tiene en cuenta el espacio que hay dentro de un objeto o figura específica; estas relaciones comprenden proximidad, separación, orden, cerramiento y continuidad. Las ideas topológicas son seguidas por las relaciones proyectivas (perspectivas con proyecciones y secciones), y euclidianas (proporciones, distancias, angulosidad, paralelismo). Para Piaget, el niño primero desarrolla el espacio topológico $\mathrm{y}$, aunque las relaciones proyectivas y euclidianas se desarrollan paralelamente, estas últimas logran un equilibrio posterior en el niño(Ochaíta, 1983). La evidencia disponible sugiere que todos los tipos de ideas geométricas parecen desarrollarse con el tiempo, cada vez más integrados y sintetizados. En el trabajo de Piaget e Inhelder (1947), los tres grandes periodos en los que el niño desarrolla el pensamiento espacial son el periodo sensoriomotor, el periodo de las operaciones concretas, que se subdivide en preoperatorio y en operaciones concretas, y periodo de las operaciones formales. Mientras que Piaget e Inhelder sugieren que el desarrollo de la percepción como se describe por los tipos de geometría es secuencial (es decir, Topológico, Proyectivo, Euclidiano), otros investigadores creen que todos los tipos de pensamiento geométrico continúan desarrollándose con el tiempo y se integran cada vez más.

Jean Piaget tuvo gran influencia en teóricos como David Ausubel y Zoltan Dienes. David Ausubel relacionó la teoría de desarrollo cognitivo por esquemas conceptuales con su explicación de cómo las personas adquieren el conocimiento. David Ausubel (Ausubel, Novak y Hanesian, 1983) teorizó que la gente adquiere un conocimiento cuando es expuesta 
Zoltan Dienes también se inspiró en la obra de Piaget y realizó experiencias que le llevaron a enunciar una teoría sobre el aprendizaje de las matemáticas y cree en enseñar y aprender matemáticas permitiendo que los niños jueguen con materiales que gradualmente introduzcan directamente a él en lugar de descubrirlo. Para Ausubel, el significado se crea a través de algunas formas de equivalencia representacional entre el lenguaje (símbolos) y el contexto mental. En el aprendizaje se involucran dos procesos fundamentales: (I) Recepción, que se emplea en el aprendizaje significativo verbal y (II) Descubrimiento, que se emplea en la formación del concepto y la resolución de problemas. Ausubel señaló que más que con el método utilizado, la recepción se vuelve más significativa cuando se usan apropiadamente diferentes técnicas de enseñanza, se emplean materiales y contenidos adecuados. Desarrollar el pensamiento matemático es uno de los principales objetivos de la educación matemática. Cada docente debe incorporar actividades que ayudarán a todos los estudiantes a progresar en el desarrollo de razonamiento analítico, razonamiento crítico, resolución de problemas y habilidades de comunicación y adquisición de hábitos matemáticos.

\section{Pensamiento matemático}

El desarrollo del pensamiento matemático resulta ser uno de los principales objetivos de la educación matemática. Cada docente debe incorporar actividades que ayudarán a todos los estudiantes a progresar en el desarrollo de razonamiento analítico, razonamiento crítico, resolución de problemas y habilidades de comunicación y adquisición de hábitos matemáticos; D’Amore (2008) reitera que los docentes para tomar decisiones en el aula explícita o implícitamente utilizan su conocimiento, método y creencia personal, sobre las formas de encontrar, aprender u organizar el conocimiento cultural. Este bagaje epistemológico llamado espontáneo que ha sido construido de forma empírica para responder a las necesidades didácticas, y hacerlos aceptar por sus alumnos y por su entorno en ocasiones les conlleva a obstáculos epistemológicos pues para enseñar matemática no sólo es necesario tener conocimiento del tema si no un manejo adecuado de su didáctica.

Teniendo como referente los trabajos de Gningue (2006) y Vasco (1984), a continuación se analizan las aportaciones teóricas de Zoltan Dienes y Carlos Eduardo Vasco Uribe.

Zoltan Dienes también se inspiró en la obra de Piaget y realizó experiencias que le llevaron a enunciar una teoría sobre el aprendizaje de las matemáticas y cree en enseñar y aprender matemáticas permitiendo que los niños jueguen con materiales que gradualmente introduzcan, expandan o consoliden conceptos matemáticos y se articule dicha enseñanza a través del trabajo en grupo, no competitivo, sino cooperativo, de modo que los problemas requieren que los estudiantes trabajen juntos, a su vez que se enseña con experiencias concretas y significativas usando materiales manipulativos. Vygotsky también sostuvo que el desarrollo psicológico individual no podía entenderse sin referencia al medio social y cultural en el que está inmerso. Los procesos mentales superiores en el individuo 
tienen su origen en procesos y contextos sociales (Espinosa \& Jiménez, 2014). En consecuencia, los procesos mentales solo pueden comprenderse si entendemos las herramientas físicas y los signos psicológicos que los median. Vygotsky (Baquero, 1996) creía que los niños tienen sus propias comprensiones y creencias matemáticas basadas en sus experiencias, pero que es la responsabilidad del adulto influir en el pensamiento del niño: las habilidades psicológicas de una persona primero se manifiestan en el ámbito social y progresivamente en la persona misma: la atención, la memoria, la formulación de conceptos son primero fenómenos sociales que se transforman en una propiedad de cada individuo, pero esta interiorización se enriquece gracias a las interacciones sociales, a la zona de desarrollo próximo: docentes, padres de familia, y compañeros son inicialmente los responsables primarios del aprendizaje, aunque sea el individuo quien potencialice sus propias capacidades. Herramientas como símbolos, palabras, diagramas, signos, la escritura, son el puente entre las habilidades interpsicológias e intrapsicológicas; son producto del contexto socio histórico.

En matemáticas, los mismos signos matemáticos median dos procesos: el desarrollo de un concepto matemático en la persona que aprende y la interacción de esa persona con el mundo matemático. Lo anterior porque la teoría de Vygotsky establece que los usos del signo son una parte necesaria de la formación de conceptos, pues forma vínculos entre actividades específicas de la matemática y la formación de esos conceptos (Radford, 2000 citado por Berger, 2005).

Por su parte, en Colombia, Carlos Eduardo Vasco, propuso el enfoque de Sistemas, en donde plantea rescatar la forma en como los niños van construyendo las matemáticas y sugiere que se vean las distintas regiones de la matemática como sistemas y no como meros conjuntos; lo anterior porque el estudiante relaciona ideas previas, situaciones propias de su entorno, manipula objetos de su entorno, entre otros, en la praxis. Por ello, para Vasco, el desarrollo del pensamiento parte de sistemas concretos, de los cuales pueden salir los sistemas conceptuales que el docente debe enseñarle a sus estudiantes, teniendo como meta llegar a la cúspide de la simbolización. La sugerencia pedagógica de Carlos Vasco es explotar los sistemas concretos que ya manejan los niños y, a partir de ellos, construir los sistemas conceptuales, así, cuando el estudiante haya iniciado su propia construcción, podrá desarrollar los sistemas simbólicos apropiados.

\section{El pensamiento geométrico}

El currículo de matemáticas en Colombia le ha dejado escaso espacio a la geometría, un fenómeno que se remonta a los años 60 cuando el álgebra comenzó a dominar el currículo (Posada, 2012). Más recientemente, esta falta de tiempo del plan de estudios para la geometría se ha visto exacerbada por un aumento sustancial en la cobertura de la estadística y aritmética (Segundo 
\& Mulett, 2014). En los lineamientos curriculares del Ministerio de Educación, la investigación moderna sobre el proceso de construcción del pensamiento geométrico indica que este sigue una evolución muy lenta desde las formas intuitivas iniciales hasta las formas deductivas finales, aunque los niveles finales corresponden a niveles escolares bastante más avanzados que los que se dan en la escuela. Por ello, se considera que el modelo de Van Hiele es la propuesta que parece describir con bastante exactitud esta evolución y que está adquiriendo cada vez mayor aceptación a nivel internacional en lo que se refiere a geometría escolar.

El modelo Van Hiele nació a partir de las observaciones que Pierre y Diana Van Hiele notaron sobre las dificultades del aprendizaje de la geometría de sus estudiantes. Estas observaciones los llevaron a desarrollar una teoría que involucra niveles de pensamiento que los estudiantes pasan a medida que progresan desde el simple reconocimiento de una figura hasta poder escribir una demostración geométrica formal.

La naturaleza sucesiva de los niveles de Van Hiele del pensamiento geométrico es una evocación de los paradigmas secuenciales de Piaget y Dienes y posee una perspectiva constructivista, en cuanto que incluye la idea de que el alumno participa activamente en la construcción de su conocimiento. El modelo de van Hiele identificó el papel del lenguaje en el aprendizaje y estableció que los estudiantes avanzan en geometría a través de niveles de pensamiento y la forma en que pasan de un nivel de comprensión al siguiente: el progreso de uno de los niveles de Van Hiele al siguiente depende más del método de enseñanza recibida que de la edad o madurez y no es posible que los estudiantes se salten un nivel. Van Hiele (Howse \& Howse, 2014) plantea cinco niveles, que son secuenciales $\mathrm{y}$ jerárquicos:

Nivel 1 (Visualización): Los estudiantes reconocen las figuras solo por apariencia, a menudo comparándolas con un prototipo conocido. No se perciben las propiedades de una figura. En este nivel, los estudiantes toman decisiones basadas en la percepción, no en el razonamiento.

Nivel 2 (Análisis): Los estudiantes ven las figuras como colecciones de propiedades. Pueden reconocer y nombrar propiedades de figuras geométricas, pero no ven relaciones entre estas propiedades. Al describir un objeto, un estudiante que opera en este nivel podría enumerar todas las propiedades que el estudiante conoce, pero no discernir qué propiedades son necesarias y cuáles son suficientes para describir el objeto.

Nivel 3 (Abstracción): Los estudiantes perciben las relaciones entre las propiedades y entre las figuras. En este nivel, los estudiantes pueden crear definiciones significativas y dar argumentos informales para justificar su razonamiento. Se entienden las implicaciones lógicas y inclusiones de clase, tales como los cuadrados que son un tipo de rectángulo. Sin embargo, el papel y el significado de la deducción formal no se entiende. 
Nivel 4 (Deducción): Los estudiantes pueden construir pruebas, entender el papel de los axiomas y las definiciones, y conocer el significado de las condiciones necesarias y suficientes. En este nivel, los estudiantes deben ser capaces de construir pruebas tales como las que se encuentran típicamente en una clase de geometría de la escuela secundaria.

Según Van Hiele, un estudiante progresa a través de cada nivel de pensamiento como resultado de la enseñanza que se organiza en cinco fases de aprendizaje que son:

Información: A través de discusión, el profesor identifica lo que los estudiantes ya saben sobre un tema y los estudiantes se orientan al nuevo tema.

Orientación guiada: Los estudiantes exploran los objetos a aprender en tareas estructuradas como doblar, medir o construir. El profesor se asegura de que los estudiantes exploren conceptos específicos.

Explicación: Los estudiantes describen en sus propias palabras lo que han aprendido sobre el tema. El profesor introduce términos matemáticos relevantes.

Libre Orientación: Los estudiantes aplican las relaciones que están aprendiendo para resolver problemas e investigan tareas terminadas.

Integración: Los estudiantes resumen e integran lo que han aprendido, desarrollando una nueva red de objetos y relaciones.

Un estudiante puede necesitar recorrer algunas de las cinco fases más de una vez con un tema en particular. Ahora bien, $\mathrm{Si}$ un docente intenta enseñar a un estudiante un nivel cuando el estudiante no ha pasado por el nivel anterior, el estudiante no entenderá al docente y recurrirá al aprendizaje memorístico, por ello, también el lenguaje juega un papel importante en el aprendizaje (Pegg, 2014): cada nivel de pensamiento tiene su propio lenguaje y su propia interpretación de un mismo término. Van Hiele y Vygotsky veían crucial el papel del maestro y el papel del lenguaje en la construcción del conocimiento por parte de un individuo: la discusión y la verbalización de conceptos entre pares y docente-estudiante, son aspectos importantes de las fases de Aprendizaje de Información, Explicación e Integración pues los estudiantes aclaran y reorganizan sus ideas al hablar de ellas.

Finalmente, desde la propuesta se hace énfasis en que el estudio de la geometría contribuye a desarrollar en los estudiantes habilidades de visualización, pensamiento crítico, intuición, perspectiva, resolución de problemas, conjetura, razonamiento deductivo, y argumento lógico. Las representaciones geométricas se pueden utilizar para ayudar a los estudiantes a dar sentido a otras áreas de la matemática: fracciones y multiplicación en aritmética, relaciones entre gráficos de funciones (de dos y tres variables), y representaciones gráficas de datos en estadística. El razonamiento espacial es importante en otras áreas curriculares, así como en las matemáticas: ciencia, geografía, arte, diseño y tecnología (Segundo \& Mulett, 2014).
Van Hiele y Vygotsky veían crucial el papel del maestro y el papel del lenguaje en la construcción del conocimiento por parte de un individuo: la discusión y la verbalización de conceptos entre pares y docente-estudiante, 


\section{Metodología}

La presente investigación sigue un enfoque cualitativo con el objetivo de tener una visión integral del pensamiento matemático y hacer una lectura holística de dicha realidad. Casilimas (2002) expresa: "los procesos de investigación cualitativa son de naturaleza multicíclica o de desarrollo en espiral y obedecen a una modalidad de diseño semiestructurado y flexible" (p. 41).

La elaboración de la propuesta se hace de la mano de la metodología de investigación acción educativa con enfoque cualitativo. La investigación acción educativa sucede cuando las personas investigan su propia práctica con el fin de mejorarla y llegar a una mejor comprensión de sus situaciones de práctica. Es acción porque actúan dentro de los sistemas que están tratando de mejorar y entender. Es una investigación porque es una investigación sistemática y crítica que se hace pública. Rahman y Fals Borda (1991), citados por Casilimas (2002), expresan que "la IAP debe verse en la actualidad 'como un puente hacia otras formas de explicación de la realidad y otras formas más satisfactorias de acción para transformarla...”' (p. 69).

John Elliot (1993) define la investigación acción como "un estudio de una situación social con el fin de mejorar la calidad de la acción dentro de la misma”. Para Elliot, la investigaciónacción es el proceso reflexivo por el cual los educadores estudian sistemáticamente sus problemas para orientar, corregir y evaluar sus decisiones y acciones en relación con la mejora de la enseñanza y el aprendizaje en su contexto profesional individual. La investigación-acción es un proceso cíclico y colaborativo de planificación estratégica, acción, implementación del plan y observación, evaluación y autoevaluación, reflexión crítica y autocrítica sobre los resultados y cambio en los sistemas sociales, culturales y políticos.

La presente propuesta se divide en tres etapas: la primera, el diagnóstico y caracterización del desempeño de los estudiantes de la Institución Educativa Técnica y Académica Antonio Nariño; una segunda etapa es la intervención de la malla curricular de matemáticas vigente para el año 2017 y una tercera etapa que consiste en la elaboración de la propuesta final, que permite contribuir al mejoramiento del pensamiento matemático desde la visión geométrica en contexto.

La segunda etapa va enfocada hacia el diseño e implementación de la estrategia. Esta fase se desarrollará en diez sesiones teniendo en cuenta la secuencia didáctica que hace parte del modelo pedagógico del colegio y que contiene diversas actividades en torno a la temática del tercer periodo correspondiente a fracciones en los tres grados focalizados, teniendo en cuenta el nivel de profundidad de cada grado.

La tercera etapa: elaboración de la propuesta final, partiendo de los resultados de la etapa dos y del análisis de las rúbricas de desempeño aplicadas, de las matrices de la observación 
participante. Cabe aclarar que la estrategia, aunque está planeada y diseñada con anterioridad es flexible dependiendo de las circunstancias educativas y las situaciones observadas en cada sesión, es decir que se van adecuando en la medida que se evalúa el trabajo desarrollado

La primera etapa parte del diagnóstico realizado a partir de los resultados obtenidos por los estudiantes de los grupos focalizados: 301, 503 y 601 en las pruebas diagnóstica, primer y segundo periodo del año 2017, así como de la observación de los docentes investigadores, lo que permite de manera concisa estructurar la caracterización del grupo de estudiantes objeto de investigación y un análisis primario de los procesos enseñanza aprendizaje llevados a cabo tradicionalmente.

De acuerdo con la experiencia como docentes de aula, se incorpora metodológicamente el uso de las TIC en la propuesta didáctica (en la medida real de las posibilidades en cada grupo y sede), mediante hardware: Tablero Interactivo, Tablet, PC, Video Beam, Tv y software aplicado de uso libre disponible en la IE.

Otro elemento que resulta esencial en la propuesta es la incorporación, adaptación, uso, evaluación y análisis de rubricas de desempeño a aplicarse durante la segunda etapa del proceso de investigación. Para Díaz Barriga (2005) citado por Martínez (2008), "las rúbricas son guías o escalas de evaluación donde se establecen niveles progresivos de dominio o pericia relativos al desempeño que una persona muestra respecto de un proceso o producción determinada" (p 129). Estas se disponen para su desarrollo a lo largo de las 10 semanas de intervención en por lo menos una por Estándar, ya sean de tipo Holístico y/o Analítico. Para ello se dispondrá de técnicas e instrumentos para su registro que obedecen a un consenso entre los docentes investigadores.

\section{Resultados}

Como resultados se tienen a la fecha, el diagnóstico de los datos institucionales sobre desempeño de estudiantes de los grados focalizados para el estudio, lo que determinó para los docentes investigadores un punto de partida para la estructuración de acciones metodológicas en la investigación.

En concordancia con los objetivos de la investigación el caracterizar la población arroja desempeños reales $\mathrm{y}$ dicientes sobre componentes y niveles de competencia de los grupos focalizados y permite explorar necesidades en cuanto a estrategias didácticas y empoderamiento de las temáticas propuestas para el diseño de la propuesta de intervención que permita mejorar dichos procesos y fortalecer el pensamiento matemático usando como herramienta estratégica la geometría contextualizada (Fase 2), posibilitando mejorar las actitudes de los estudiantes frente al aprendizaje de las matemáticas. 
Tabla 1

Resultados prueba diagnóstica, primer y segundo periodo grado 301

\begin{tabular}{|c|c|c|c|c|c|c|c|}
\hline & Competencias & Diagnóstica & Nivel desempeño & $1^{\circ}$ periodo & Nivel desempeño & $2^{\circ}$ periodo & $\begin{array}{c}\text { Nivel } \\
\text { desempeño }\end{array}$ \\
\hline $\mathrm{C} 1$ & $\begin{array}{l}\text { Razonamiento y } \\
\text { argumentación }\end{array}$ & 7,30 & III & 3,03 & II & 3,87 & II \\
\hline $\mathrm{C} 2$ & $\begin{array}{l}\text { Comunicación, } \\
\text { representación y } \\
\text { modelación }\end{array}$ & 6,51 & II & 5,92 & II & 5,48 & II \\
\hline C3 & $\begin{array}{l}\text { Planteamiento y } \\
\text { resolución de problemas }\end{array}$ & 7,31 & III & 6,06 & II & 2,18 & I \\
\hline & Componentes & Diagnóstica & Nivel desempeño & $1^{\circ}$ periodo & Nivel desempeño & $2^{\circ}$ periodo & $\begin{array}{c}\text { Nivel de } \\
\text { desempeño }\end{array}$ \\
\hline $\mathrm{C} 1$ & Numérico - variacional & 7,07 & A & 5,95 & M & 2,42 & B \\
\hline $\mathrm{C} 2$ & Geométrico- métrico & 4,64 & M & 4,85 & M & 5,59 & M \\
\hline $\mathrm{C} 3$ & Aleatorio & 8,19 & A & 7,73 & A & 3,39 & M \\
\hline
\end{tabular}

Tabla 2

Resultados prueba diagnóstica, primer y segundo periodo grado 503

\begin{tabular}{|c|c|c|c|c|c|c|c|}
\hline & Competencias & Diagnóstica & $\begin{array}{c}\text { Nivel } \\
\text { desempeño }\end{array}$ & $1^{\circ}$ periodo & $\begin{array}{l}\text { Nivel } \\
\text { desempeño }\end{array}$ & $2^{\circ}$ periodo & $\begin{array}{l}\text { Nivel } \\
\text { desempeño }\end{array}$ \\
\hline $\mathrm{C} 1$ & $\begin{array}{l}\text { Razonamiento y } \\
\text { argumentación }\end{array}$ & 6,92 & II & 7,71 & III & 7,42 & III \\
\hline $\mathrm{C} 2$ & $\begin{array}{l}\text { Comunicación, } \\
\text { representación y } \\
\text { modelación }\end{array}$ & 6,13 & II & 8,26 & III & 7,85 & II \\
\hline C3 & $\begin{array}{l}\text { Planteamiento } \\
\text { y resolución de } \\
\text { problemas }\end{array}$ & 4,89 & II & 7,06 & III & 6,41 & III \\
\hline & Componentes & Diagnóstica & $\begin{array}{c}\text { Nivel } \\
\text { desempeño }\end{array}$ & $1^{\circ}$ periodo & $\begin{array}{c}\text { Nivel } \\
\text { desempeño }\end{array}$ & $2^{\circ}$ periodo & $\begin{array}{c}\text { Nivel de } \\
\text { desempeño }\end{array}$ \\
\hline $\mathrm{C} 1$ & $\begin{array}{l}\text { Numérico - } \\
\text { variacional }\end{array}$ & 5.44 & M & 7.71 & A & 7.58 & A \\
\hline $\mathrm{C} 2$ & $\begin{array}{l}\text { Geométrico- } \\
\text { métrico }\end{array}$ & & & 7.29 & A & 7.88 & A \\
\hline $\mathrm{C} 3$ & Aleatorio & 6.07 & M & 8.02 & A & 7.21 & A \\
\hline
\end{tabular}

Tabla 3

Resultados prueba diagnóstica, primer y segundo periodo grado 503

\begin{tabular}{|c|c|c|c|c|c|c|c|}
\hline Competencias & Diagnóstica & $\begin{array}{c}\text { Nivel } \\
\text { desempeño }\end{array}$ & $1^{\circ}$ periodo & $\begin{array}{c}\text { Nivel } \\
\text { desempeño }\end{array}$ & $2^{\circ}$ periodo & $\begin{array}{c}\text { Nivel } \\
\text { desempeño }\end{array}$ \\
\hline C1 & $\begin{array}{l}\text { Razonamiento y } \\
\text { argumentación }\end{array}$ & 3,82 & II & 5,05 & II & 7,36 & III \\
\hline $\begin{array}{l}\text { Comunicación, } \\
\text { representación y } \\
\text { modelación }\end{array}$ & 6,88 & II & 6,31 & II & 6,07 & II \\
\hline $\begin{array}{l}\text { Planteamiento } \\
\text { y resolución de } \\
\text { problemas }\end{array}$ & 6,83 & II & 5,86 & II & 5,12 & II \\
\hline
\end{tabular}




\begin{tabular}{|l|l|c|c|c|c|c|c|}
\hline & Componentes & Diagnóstica & $\begin{array}{c}\text { Nivel } \\
\text { desempeño }\end{array}$ & $\mathbf{1}^{\circ}$ periodo & $\begin{array}{c}\text { Nivel } \\
\text { desempeño }\end{array}$ & $\mathbf{2}^{\circ}$ periodo & $\begin{array}{c}\text { Nivel de } \\
\text { desempeño }\end{array}$ \\
\hline C1 & $\begin{array}{l}\text { Numérico } \\
\text { variacional }\end{array}$ & 5,97 & $\mathrm{M}$ & 6,43 & $\mathrm{M}$ & $\mathbf{4 , 6 6}$ & $\mathrm{M}$ \\
$\mathrm{C} 2$ & $\begin{array}{l}\text { Geométrico- } \\
\text { métrico }\end{array}$ & 5,83 & $\mathrm{M}$ & 5,14 & $\mathrm{M}$ & 5,89 & $\mathrm{M}$ \\
$\mathrm{C} 3$ & Aleatorio & 6,71 & $\mathrm{M}$ & 6,15 & $\mathrm{M}$ & 7,99 & $\mathrm{~A}$ \\
\hline
\end{tabular}

Los resultados obtenidos en las tres pruebas, diagnóstica, primer y segundo periodo, se presentan en las tablas 1, 2 y 3 , los cuales permiten estructurar la fase de intervención.

La Tabla 1 presenta los resultados del grado 301. Para la prueba diagnóstica el componente geométrico y métrico es el de menor valoración con un 4,64 observando que no se obtiene los resultados esperados. También se observan en la competencia de representación y modelación con una valoración de 6,51. De igual manera, para el primer periodo se observa que el componente con menor valoración es el geométrico y métrico con una valoración de 4,85 pero en esta ocasión la competencia con menor valoración es la de razonamiento y argumentación con 3,03, todos situados en un desempeño medio; para el segundo periodo es notorio que el componente numérico variacional está en 2,42 ubicado en un desempeño bajo; el componente aleatorio con 3,39 y geométrico y métrico con 5,59 , ubicados en un desempeño medio; por otra parte, las competencias planteamiento y resolución de problemas con 2,18, razonamiento y argumentación con 3,87 , y comunicación y representación 5,48 , ubicándose en un desempeño medio; los anteriores resultados responden al abordaje previo de diferentes contenidos propuestos en las mallas curriculares de la I.E en el año 2016 para la diagnóstica y primer y segundo periodo de 2017; sin embargo, se presume que el nivel de dificultad de los contenidos a medida que se avanza es mayor, por tal razón se presenta una disminución del puntaje al trascurrir del primer y segundo periodo y por su puesto las didácticas utilizadas para estos periodos son diferentes pero no responden a las pretensiones de la institución. También se observa que en actividades de retroalimentación de las mismas pruebas hay dificultades y responden a las competencias de razonamiento argumentación, planteamiento y resolución de problemas y comunicación, modelación y representación, pues la mayor oportunidad de mejoramiento está en la comprensión de la lectura e interpretación de la misma, dando como resultado confusión en las respuestas y contestando erróneamente, obteniendo los resultados anteriormente expuestos.

Para el grado 503, Tabla 2, la prueba diagnóstica presenta los puntajes más bajos en las tres aplicaciones, y en ella solo se evaluaron componente $\mathrm{C} 1$ y C3 (no componente Geométrico- métrico), concluyendo que el proceso durante el trascurso del año ha sido efectivo. 
Los diferentes resultados variaron durante las tres aplicaciones, aunque el componente geométrico métrico mantuvo su tendencia creciente en las pruebas de primer y segundo periodo convirtiéndose en la fortaleza que permite potenciar los demás componentes.

El componente aleatorio muestra fluctuaciones de casi un punto en las tres aplicaciones, creciendo en el primer periodo con respecto a la prueba diagnóstica y decreciendo nuevamente en el segundo periodo. Se concluye que a los estudiantes se les dificultaron los conceptos de media, moda y mediana, temática trabajada durante este periodo.

La competencia que más genera dificultad es la de planteamiento y resolución de problemas en las tres aplicaciones, presentando los puntajes más bajos a pesar de haber crecido en 2,17 puntos entre la prueba diagnóstica y la prueba del primer periodo; sin embargo, en la aplicación de segundo periodo volvió a decrecer. Se concluye que hay dificultades en la comprensión lectora, aspecto que se debe fortalecer. Durante las realimentaciones de las pruebas realizadas se les facilitó a los niños trabajar con material manipulativo para resolver algunas de las preguntas, permitiéndoles así investigar y jugar con formas geométricas para que pudiesen identificar las propiedades de las figuras y reconocerlas por sus propiedades, en lugar de confiar en su apariencia (segundo nivel de van Hiele (análisis)).
Eldiagnóstico en el grupo focalizado 601 desde el punto de vista del desempeño general en las pruebas validadas "instruimos 2017": Diagnóstica, 1er periodo y 2 do periodo sitúan al grupo en general en un nivel de desempeño II (Medio) con una tendencia próxima al nivel III (Alto), Tabla 3. Esto determina para los investigadores un adecuado punto de partida para el diseño, implementación, valoración y análisis de la propuesta de intervención (Fase II) de la Investigación.

Específicamente analizando las competencias evaluadas: C1: Razonamiento y argumentación; C2: Comunicación, representación y modelación; y, C3: Planteamiento y resolución de problemas, se observa una tendencia creciente en la primera, una tendencia constante en la segunda, y una tendencia decreciente en la tercera, lo que puede entenderse como una alerta para reforzar desde la práctica docente dicha competencia (con la geometría como elemento integrador) que resulta ser un objetivo de la investigación.

Lo anterior reafirma, en primera instancia, la esencia del método de investigación acción educativa abordado en la investigación (Elliot 2000); y en segundo término, la necesidad de diseñar una propuesta metodológica de aula a fin de mejorar los niveles de desempeño, la competencia en sí misma, y en general, el pensamiento matemático de los grupos focalizados a partir de la reflexión, la observación directa y la implementación de estrategias activas en aula. 


\section{Conclusiones}

La geometría como eje integrador del pensamiento matemático en educación básica es una propuesta que invita al docente a reflexionar y actuar efectivamente sobre las interacciones que se dan en el aula, las motivaciones que traen los niños a la institución educativa, las propias prácticas de los docentes y sobre todo la interacción de los contenidos en ese mundo diferencial y único. Partiendo del modelo Van Hiele, el uso de la geometría como herramienta didáctica para la enseñanza de la matemática básica no es apenas sino el asomo para buscar una verdadera transformación de la escuela, una incluyente y formadora en nuestro sistema educativo y la contribución e invitación al cambio desde la práctica docente; la geometría es quizás la parte del plan de estudios de la matemática en donde es posible tener más diversión: es visual, intuitiva, creativa y exigente.

De igual forma, el uso de recursos concretos permite la oportunidad para el juego informal, que es apoyado por la teoría Zoltan Dienes. Esto debe tener lugar al principio de todo aprendizaje, ya que da a los estudiantes la oportunidad de investigar un concepto primero y luego hacer conexiones cuando los métodos formales se introducen a través de la enseñanza; también le permite familiarizarse con los recursos y lo que representan.

La realización de investigaciones en el campo de la educación matemática permiten establecer la comprensión de conceptos puntuales y trascendentes y abren el camino para la proposición e implementación de modelos pedagógicos útiles para el aprendizaje y la didáctica de las matemáticas e invita al docente a reflexionar y actuar efectivamente sobre las interacciones que se dan en el aula (método de investigación acción educativa (Elliot, 2000)).

Finalmente, en cuanto al presente trabajo de investigación, se ha empezado a trabajar en al fase dos: diseñar la propuesta metodológica basada en las mallas curriculares manejadas en la institución. Se han trabajado hasta el momento seis sesiones con los estudiantes de los grados focalizados, en donde se ha aplicado una rúbrica holística para trabajo en grupo y rúbricas analíticas por temática (tres en total), los docentes investigadores han participado en la elaboración, apliación y análisis de las sesiones. De igual forma se puede decir que el índice de reprobación en los estudiantes de los grupos focalizados ha disminuido y el rendimiento académico se ha incrementado en el área. 


\section{Referencias}

Álvarez-Gayou, J. L. (2003). Cómo hacer investigación cualitativa. Fundamentos y metodología. Cáp. I. Colección Paidós Educador. México: Paidós Mexicana.

Arias, F. (2006) El Proyecto de Investigación. Introducción a la Investigación Científica. Quinta edición. Editorial Episteme.

Baquero, R. (1996) Vigotsky y el aprendizaje escolar. Buenos Aires: Aique

Bizquera, (1990) Métodos de Investigación Educativa. España: Editorial CEAC.

Camargo, L. (2012) La geometría, su enseñanza y su aprendizaje. Tecné, Episteme y Didaxis, (32), 4-8.

D' amore, B. (2011). Epistemology, didactics of mathematics and teaching practices. Mediterranean Journal for Research in Mathematics Education, 7(1), 1-22.

Elliot, J. (2000). La investigación-acción en educación. Ediciones Morata.

Espinosa, C., \& Jiménez, A. (2014). Construcción del concepto de razón y razón constante desde la óptica socioepistemológica. Praxis \& Saber, 5(9), 53-80. https://doi.org/10.19053/22160159.2993

Gningue, S. (2006). Students Working Within and Between Representations: An Application of Dienes's Variability Principles. For the Learning of Mathematics, 26(2), 41-47.

. (2016). Remembering Zoltan Dienes, a Maverick of Mathematics Teaching and Learning: Applying the Variability Principles to Teach Algebra. International Journal For Mathematics Teaching and Learning, 17(2), 1-24

Gutiérrez, Á., \& Jaime, A. (1998). Geometría y algunos aspectos generales de la Educación Matemáticas.

Gutiérrez, Á. (2011). Reflexiones sobre la enseñanza de la geometría en los niveles de primaria y secundaria. En Perry, P. (Ed.), Memorias 20 Encuentro de Geometría y sus Aplicaciones (pp. 3-14). Bogotá, Colombia: Universidad Pedagógica Nacional.

Howse, T. D., \& Howse, M. E. (2014). Linking the van Hiele Theory to Instruction. Teaching Children Mathematics, 21(5), 304-313.

Hurtado, J. (2000). Metodología de la investigación. Caracas: Holística Fundación SYPAL.

Martínez-Rojas, J. G. (2008). Las rúbricas en la evaluación escolar: su construcción y su uso. Avances en medición, 6(129), 38. 
Ministerio de Educación Nacional. (2006). Estándares Básicos de Competencias en Matemáticas. Estándares Básicos de Competencias En Lenguaje, Matemáticas, Ciencias Y Cuidadanas, 46-95

Ochaíta, E. (1983). La teoría de Piaget sobre el desarrollo del conocimiento espacial. Estudios de Psicología, (14-15), 93-108.

Pegg, J. (2014). The van Hiele Theory. Encyclopedia of Mathematics Education, 26-59. https://doi.org/10.1007/978-94-007-4978-8_183

Piedrahita, W., Londoño, J., \& Uribe, E. (2009) La enseñanza de la geometría con fundamento en la solución de problemas cotidianos: una propuesta metodológica orientada por el modelo de Van Hiele, aplicada a estudiantes de la I.E. Rafael J. Mejia del municipio de Sabaneta (Ant.) Tesis (Maestría en Educación y Desarrollo Humano). Universidad de Manizales. Facultad de Ciencias Sociales y Humanas. CINDE,

Posada, S. (2012). La geometría en el aula "Una propuesta para la interpretación de conceptos e ideas matemáticas y físicas”, 1209-1214.

Sabino, C. (1992) El Proceso de Investigación. Bogotá: Editorial Panamericana.

Sandoval, C. (2002). Módulo 4: Investigación cualitativa. Medellín: INER-Universidad de Antioquia.

Segundo, A., \& Mulett, G. (2014). Historia Social de la Educación Matemática en Iberoamérica: Cincuenta años de reformas en el currículo colombiano de Matemática en los niveles básico y medio de educación. Revista Iberoamericana de Educación Matemática, 155-176.

Sierra R. (1998) Técnicas de investigación social: teoría y ejercicios (10a ed.). Madrid: Editorial Paraninfo.

Tamayo y Tamayo, M. (2004). El modelo de Investigación. En El proceso de la investigación científica (3a ed) (pp. 111-141). México: Limusa. . (2007). El proceso de la investigación científica. Editorial Limusa.

Van Hiele, P. M. (2000). Begin with Play. Teaching Children Mathematics, 6 (February 1999)

Vasco, C. E. (1984). El enfoque de sistemas en el nuevo programa de matemáticas, 45-51.

- (1990). El aprendizaje de las matemáticas elementales como proceso condicionado por la cultura. CL \& E: Comunicación, Lenguaje Y Educación, 5-2 